\title{
QUALITY INSPECTION OF SATELLITE IMAGERY BLOCK ADJUSTMENT WITHOUT GCP
}

\author{
Y. Zhao ${ }^{1, *}$, H. T. Zhao ${ }^{1}$, J. Guo ${ }^{1}$, H. J. Tu ${ }^{1}$, S. Yin ${ }^{1}$ \\ ${ }^{1}$ National Quality Inspection and Testing Center for Surveying and Mapping Products, China - cloudingshinezy@163.com
}

Commission III, TCIII/IVb

KEY WORDS: Without GCP, Block Adjustment, Inspection Content, Inspection Method

\begin{abstract}
:
The current status of insufficient research on production technology and quality inspection methods for uncontrolled mapping of block adjustment results for global project. This paper proposes a set of quality inspection process and technical methods for largescale production, lack of field checkpoints and difficult to verify in the field based on the analysis of the characteristics of the results: First, analyze the quality elements and inspection contents to be inspected, determine the inspection methods for different inspection contents; Then, extract items that can be automatically checked or need to be verified the production process data, establish inspection rules, implement batch automatic inspection and production data verification for some check items; study the inspection methods for the remaining items and implement human-computer interaction check. Finally, 4 test-fields were checked and the results showed that the technical route proposed in this paper is feasible, and the quality evaluation results are objective and reliable.
\end{abstract}

\section{INTRODUCTION}

The global project which is mainly for the construction and maintenance of global geographic information resources. Block adjustment is one of the key results of global projects. Compared with the traditional block adjustment, global project block adjustment is based on China's independent highresolution remote sensing satellite as the main data source, using high-precision mapping technology to build a large-scale block without GCP. Traditional quality inspection methods have been unable to meet the requirements for the inspection of block adjustment for large-scale and without GCP. The global project block adjustment results mainly have three characteristics. First, Wide range: The global project plans to complete the construction of about 60 million square kilometers of high-resolution data resources along the Belt and Road and key African regions from 2016 to 2020, the construction of 149 million square kilometers of global geographic information resources will be completed until 2025 . Second, high quality requirements: The global project is to carry out large-scale production of DOM, DEM, DSM, and core vector products on the basis of block adjustment without GCP, therefore, the block adjustment results produced by mapping without GCP technology are the production basis for all the results of the entire global project, the quality of block adjustment is the quality basis of all results.Third, difficult to obtain field checkpoints: The main production scope of global projects is abroad, and the production measurement area lacks field points and it is difficult for field inspections.By analyzing the characteristics of the block adjustment of the global project, this paper puts forward a set of technical methods and a process for quality inspection which is suitable for large-scale production and lacking field inspection points based on the actual inspection.

\section{CONTENTS AND METHODS OF INSPECTION}

The quality inspection of global project block adjustment includes 3 quality elements that are data quality, layout quality and attachment quality, 13 inspection contents are involved.The detailed inspection contents and methods are shown in Table 1.

In order to carry out the inspection work on the block adjustment of the global project successfully,this paper adopts a combination of detailed inspection and general inspection. Detailed inspection covers all inspection samples, and general inspection covers all production results.First, use the inspection program to conduct an overview of all block adjustments, and then determine the inspection samples from all block adjustments for detailed inspection. The global project block adjustment quality inspection technical route is shown in Figure 1.

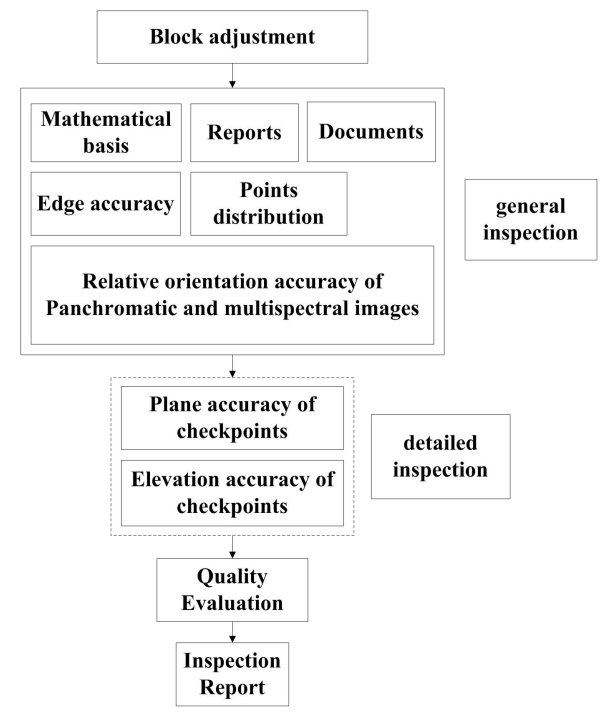

Figure 1. Block adjustment quality inspection technical route

\footnotetext{
* Corresponding author
} 


\begin{tabular}{|l|l|l|}
\hline Item & \multicolumn{1}{|c|}{ Content } & \multicolumn{1}{c|}{ Method } \\
\hline Mathematical basis & $\begin{array}{l}\text { Coordinate system , Elevation datum , Elevation } \\
\text { datum } \\
\text { Plane accuracy of checkpoint, } \\
\text { Relative orientation accuracy of Panchromatic and } \\
\text { multispectral images }\end{array}$ & $\begin{array}{l}\text { Verification of process data } \\
\text { Verification with high-precision data } \\
\text { Verification with the same accuracy data } \\
\text { Recalculation with other software } \\
\text { Verification with high-precision data } \\
\text { Verification with the same accuracy data } \\
\text { Recalculation with other software }\end{array}$ \\
Height accuracy & $\begin{array}{l}\text { Elevation accuracy of checkpoint } \\
\text { Relative orientation accuracy of block } \\
\text { Edjge accuracy } \\
\text { block adjustments } \\
\text { Relative orientation accuracy report, } \\
\text { Repolute orientation accuracy report } \\
\text { The number of connection points , } \\
\text { Connection points distribution } \\
\text { Completeness and standardization of Documents }\end{array}$ & Automatic inspection with program \\
Points distribution \\
Documents
\end{tabular}

Table 1. Inspection contents and methods

\subsection{General inspection}

The general inspection includes the inspection of relative orientation accuracy within block adjustment and the verification of key technologies in the production process of the block adjustment.

2.1.1 Inspection of relative orientation accuracy: There are clear norm requirements of relative orientation accuracy for the block adjustment in .global project. Use inspection procedures to complete inspections quickly and in batches, which only need to set the correct indicators, check content and data source,It can automatically complete the error check in the combination of each pair of panchromatic and multispectral images, the check of the edge tolerance of each pair of images between the block adjustment, and the check of the edge tolerance of each pair of adjacent images within the block adjustment.

The tolerance of panchromatic image and multi-spectral image should not be greater than 1 pixel of the multi-spectral image. The tolerance between block adjustments should be less than twice the medium error value specified in Table 3, including the plane accuracy and elevation accuracy.The tolerance of adjacent overlapping images within the block adjustment should be less than $2 \sqrt{2}$ times of the medium error value specified in Table 3 .

\begin{tabular}{|l|c|c|}
\hline Content & \multicolumn{2}{|c|}{ Norm(unit: m) } \\
\hline $\begin{array}{l}\text { Relative orientation accuracy of } \\
\text { panchromatic and multispectral } \\
\text { images }\end{array}$ & 6 & height \\
$\begin{array}{l}\text { Relative orientation accuracy of } \\
\text { block adjustments }\end{array}$ & 20 & 12 \\
$\begin{array}{l}\text { Relative orientation accuracy } \\
\text { within block adjustments }\end{array}$ & 14.1 & 8.4 \\
\hline
\end{tabular}

Table 2. Norm requirements of relative orientation accuracy

2.1.2 Verification of process data: The verification of the key technologies in the production process of the global project block adjustment can be summarized into 4 aspects: (1) The spatial reference of the results should be the WGS- 84 geodetic coordinate system, the elevation datum of the geodetic height, and the UTM projection and the polar position projection according to different regional distributions. (2) The median error of the connection points cannot exceed 0.5 pixels, the maximum error cannot exceed 1.5 pixels, and the number of connection points between 1 and 1.5 pixels cannot exceed $5 \%$ of the total number of connection points. (3) The number of connected points in the image range of each scene is in principle no less than 100 and uniformly distributed. (4) Documents such as professional technical design inspection, reports and technical summary must be comprehensive and standardized.

\subsection{Detailed inspection}

The detailed inspection content is the plane accuracy and elevation accuracy of the results. collect 20-50 checkpoints with uniform distribution and obvious characteristics in each sample, calculate the medium and maximum errors to verify the plane accuracy and elevation accuracy of the block adjustment.At the same time, the number of gross errors and gross error rate are counted to evaluate whether the position accuracy of the block adjustment meets the requirements of the global project.

\begin{tabular}{|l|c|}
\hline Content & Norm(unit: $\mathrm{m}$ ) \\
\hline Plane accuracy & 10 \\
Height accuracy & 6 \\
\hline
\end{tabular}

Table 3. Norm requirements of absolute orientation accuracy

Depending on the source of the checkpoints, there are 3 methods, such as verification with high-precision data, verification with the same accuracy data, recalculation with other software. Different calculation formulas are used to calculate the errors in plane accuracy and elevation accuracy. When using high-precision data to verify accuracy of block adjustment, the error value within 2 times (including 2 times) of the allowable medium error should participate in the accuracy statistics, and errors exceeding 2 times of the allowable medium error are regarded as gross errors. If the checkpoints are from the same accuracy data or the recalculation results, error values within $2 \sqrt{2}$ times (including $2 \sqrt{2}$ times) of the allowable medium error should participate 
in the accuracy statistics, and the error exceeds the allowable error of $2 \sqrt{2}$ times Treat as gross error.Gross error rate should be less than $5 \%$

$$
\mathrm{M}_{1}= \pm \sqrt{\frac{\sum_{i=1}^{n} \Delta_{i}^{2}}{n}} \quad ; \quad \mathrm{M}_{2}= \pm \sqrt{\frac{\sum_{i=1}^{n} \Delta_{i}^{2}}{2 n}}
$$

where $\mathrm{M}_{1}=$ medium error of verification with highprecision data

$\mathrm{M}_{2}=$ medium error of verification with the same accuracy precision data

$\mathrm{n}=$ number of checkpoints

$\Delta_{\mathrm{i}}=\mathrm{D}$-value

where $\quad \mathrm{r}=$ gross error rate

$$
r=\mathrm{n} / \mathrm{N} * 100 \%
$$

$\mathrm{n}=$ number of gross error

$\mathrm{N}=$ number of checkpoints

When the number of checkpoints is less than 20, the average value is used to replace the medium error.

2.2.1 Verification with high-precision data: The checkpoints of the high-precision inspection data fixed-point verification method are mainly derived from commercial highresolution satellite images. The checkpoints used for the quality inspection of block adjustment for global project are mainly selected from WorldView satellite images. WorldView satellite is Digitalglobe's commercial imaging satellite system. At present, it is a satellite with high spatial resolution and positioning accuracy. It can receive panchromatic and multispectral images with a spatial resolution of $0.31 \mathrm{~m}$ and a nominal positioning accuracy of 3.5-4 Meters, the accuracy meets the requirements of global project accuracy inspection data. WorldView satellite images include stereoscopic images and plane images. Stereoscopic images can be used to verify the plane accuracy and elevation accuracy of block adjustment . Plane images can only be used to verify the plane accuracy of block adjustment.

The collected WorldView satellite images generally have a range of about 25 square kilometers per piece. In order to ensure that the number and distribution of inspection points meet the requirements and have a certain representativeness, generally 3 inspection points are selected on each WorldView satellite image. Therefore, if there are more than 7 WorldView satellite image distributions in the test area, the test area can be verified by the method of verification with high-precision data. If the number of WorldView satellite images in the test field is few, then, 3 checkpoints are collected on each WorldView satellite image to calculate the location accuracy of the test field, at the same time, collected a sufficient number of checkpoints in the entire test field to verify the accuracy by other methods, such as verification with the same accuracy data, Recalculation with other software.

2.2.2 Verification with the same accuracy data: The checkpoints of the reference cross-validation method are mainly derived from public high-resolution satellite imagery. The Google Earth imagery is mainly used for quality inspection of block adjustment for global project which is a combination of satellite imagery and aerial data.The satellite image part comes from the QuickBird commercial satellite of Digital
Globe in the United States and the EarthSat company in the United States (most of the images are from the land satellite LANDSAT-7 satellite). The source of the aerial part is the British Blue Sky company (mainly for aerial photography and GIS / GPS related services), American Sanborn (mainly engaged in GIS geographic data and aerial surveys), American IKONOS and French SPOT5. After experimental verification, there are some differences in the accuracy of the Google Earth satellite image in different regions, but the plane accuracy is 5 meters and the elevation accuracy is 5 meters basically.

2.2.3 recalculation with other software: The software that produced the regional network adjustment results of the global project is pixel factory and PixGrid ,This paper proposes to use non-production software to carry out recalculation verification on the results.Using the same data source and reference data that be used in the production of results by other software to produce a repeated calculation results. Collect 20-50 checkpoints with uniform distribution and obvious characteristics both on the repeated calculation results and the original results,calculate the errors in the plane and the errors in the elevation.

\section{TEST}

\subsection{Test fields}

This paper selected 4 test areas in the world, field 1 (Tunisia, Algeria), field 2 (Israel, Syria), field 3 (western Kazakhstan), field 4 (Kenya, Tanzania). Comprehensively considering the distribution of WorldView images in each test field, different inspection methods are adopted for the plane accuracy and elevation accuracy of each block adjustment. At the same time, the relative orientation accuracy of each block adjustment is automatically checked in batches by the software and the key process data of all block adjustments are comprehensively checked. There are sufficient number of stereoscopic WorldView satellite images in field 1, a total of 41 stereoscopic images; There are sufficient number of stereoscopic WorldView satellite images in field 2, a total of 9 stereoscopic images; There are a small amount of plane WorldView satellite images in field 3, a total of 3 plane images; There are no WorldView satellite images in field 4.

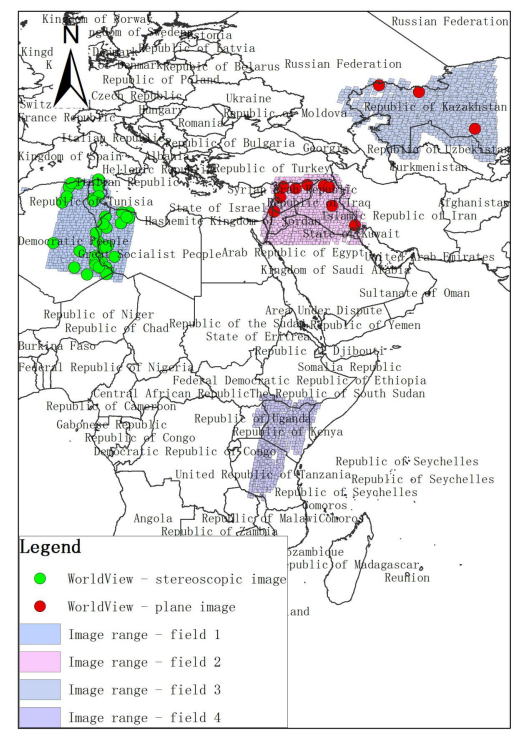

Figure 2. Distribution of WorldView image 


\begin{tabular}{|l|l|l|c|}
\hline Field & \multicolumn{2}{|c|}{ Method } & $\begin{array}{c}\text { WorldView satellite } \\
\text { images distribution }\end{array}$ \\
\hline \multirow{3}{*}{$\begin{array}{c}\text { plane } \\
2\end{array}$} & $\begin{array}{l}\text { Verification with high-precision data } \\
\text { Verification with high-precision data } \\
\text { Recalculation with other software }\end{array}$ & $\begin{array}{l}\text { Verification with high-precision data } \\
\text { Verification with the same accuracy data } \\
\text { Recalculation with other software }\end{array}$ & $\begin{array}{c}41 \text { stereoscopic images } \\
9 \text { stereoscopic images }\end{array}$ \\
& $\begin{array}{l}\text { Verification with high-precision data } \\
4\end{array}$ & $\begin{array}{l}\text { Verification with the same accuracy data } \\
\text { Verification with the same accuracy data }\end{array}$ & 3 plane images \\
\hline
\end{tabular}

Table 4. Methods of test fields

\subsection{Test results}

\subsubsection{Results of general inspection}

The spatial reference of all the 4 fields were the WGS- 84 geodetic coordinate system, the elevation datum of the geodetic height and the UTM projection. The medium errors of the connection points did not exceed 0.5 pixels, the maximum errors did not exceed 1.5 pixels, and the number of connection points between 1 and 1.5 pixels also did not exceed $5 \%$ of the total number of connection points. The number of connected points in the image range of each scene is in principle no less than 100 and uniformly distributed, except the one that most of the images are sea.Documents include professional technical design inspection, reports and technical summary all be comprehensive and standardized.

The tolerance of panchromatic image and multi-spectral image within 4 fields are mostly less than 1 pixel of the multi-spectral imag. The tolerance of adjacent overlapping images within 4 fields are mostly less than $2 \sqrt{2}$ times of the medium error value specified in Table 3.Field 3 didnot have a good result, some tolerance of each pair of images within field 3 are greater than $2 \sqrt{2}$ times of the medium error value specified in Table 3 .

\subsubsection{Results of detailed inspection}

Field 1: There are sufficient WorldView satellite stereoscopic images in field 1 . Therefore, the plane accuracy and elevation accuracy of the test area can be verified by the high-precision data. The statistical results are shown in Table 5.

The inspection results by using high-precision data show that the medium error is 3.33 meters in planimetric and 2.69 meters in height direction, maximum error is 8.94 meters in planimetric and 7.39 meters in height direction. The number of gross errors and the rate are both 0 . So, The accuracy of field 1 can meet the requirements of global project.

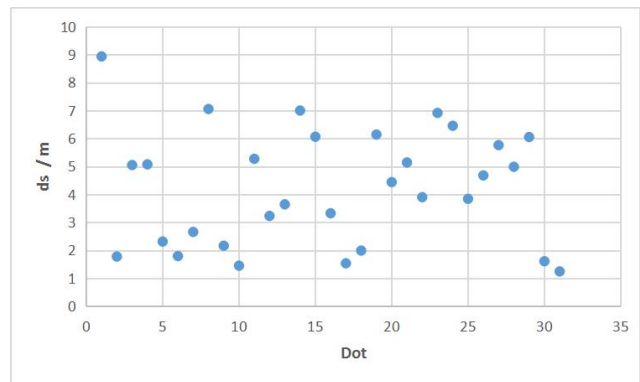

Figure 3. Errors of plane accuracy in field 1

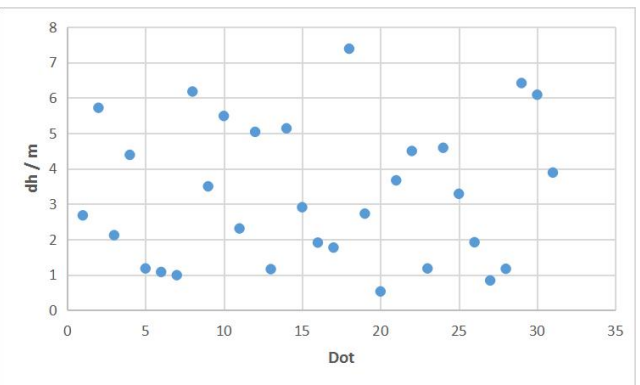

Figure 4. Errors of elevation accuracy in field 1

\begin{tabular}{|l|l|l|}
\hline & plane & height \\
\hline medium error & 3.33 & 2.69 \\
maximum error & 8.94 & 7.39 \\
$\begin{array}{l}\text { Number of gross } \\
\text { error }\end{array}$ & 0 & 0 \\
$\begin{array}{l}\text { Rate of gross error } \\
\text { method }\end{array}$ & 0 & 0 \\
\hline
\end{tabular}

Table 5. Statistical results of field 1

Field 2: There are sufficient plane WorldView satellite images in field 2. Therefore, the plane accuracy of the test area was verified by the high-precision data, the elevation accuracy of the test area was verified by the same accuracy data. Also, this paper selected the method of other software recalculation to test the plane accuracy and elevation accuracy of the field 2 . The statistical results are shown in Table 6 .

The experimental results show that, in terms of plane accuracy, the results obtained by the high-precision data detection method and other software recalculation are basically the same. The medium errors are 6.22 meters and 5.28 meters, the maximum errors are 17.67 meters and 14.44 meters. There are only 1 gross error with both methods and the gross error rate is $3.3 \%$. In terms of elevation accuracy, the results obtained by the same accuracy data detection method and other software recalculation are basically the same. The medium errors are 1.92 meters and 2.84 meters, the maximum errors are 6.78 meters and 9.36 meters. The number of gross errors and the rate are both 0 . 


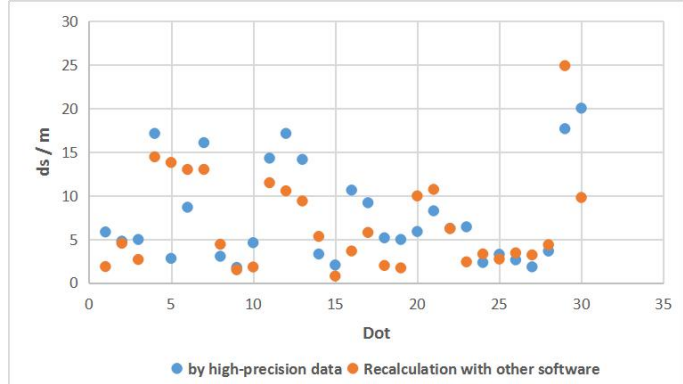

Figure 5. Errors of plane accuracy in field 2

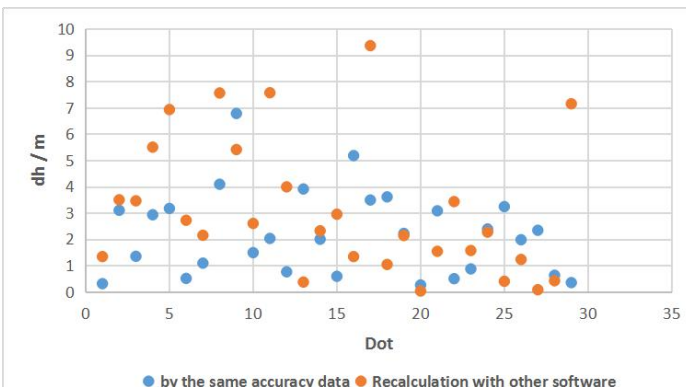

Figure 6. Errors of elevation accuracy in field 2

\begin{tabular}{|c|c|c|c|c|}
\hline & plane & plane & height & height \\
\hline \multirow{5}{*}{$\begin{array}{l}\text { medium } \\
\text { error } \\
\text { maximum } \\
\text { error } \\
\text { Number of } \\
\text { gross error } \\
\text { Rate of } \\
\text { gross error } \\
\text { method }\end{array}$} & 6.22 & 5.28 & 1.92 & 2.84 \\
\hline & 17.67 & 14.44 & 6.78 & 9.36 \\
\hline & 1 & 1 & 0 & 0 \\
\hline & $3.3 \%$ & $3.3 \%$ & 0 & 0 \\
\hline & $\begin{array}{l}\text { high- } \\
\text { precision } \\
\text { data }\end{array}$ & recalculation & $\begin{array}{l}\text { the same } \\
\text { accuracy } \\
\text { data }\end{array}$ & recalculation \\
\hline
\end{tabular}

Table 6. Statistical results of field 2

Field 3: There is only a small amount of plane WorldView satellite images in field 3 . Therefore, the plane accuracy of the test area is verified by the high-precision data. This paper also used the same accuracy data to check the plane accuracy of field 3. In terms of elevation accuracy, there is no highprecision data and the method of the same accuracy data verification was used for inspection. The statistical results are shown in Table 7.

The experimental results show that, in terms of plane accuracy, the results obtained by the high-precision data detection method and the same accuracy are basically the same. The medium errors are 15.7 meters and 7.14 meters, the maximum errors are 18.25 meters and 16.85 meters. The number of gross errors are 3 and 2, the gross error rates are $37.5 \%$ and $8.7 \%$. In terms of elevation accuracy, the medium error is 2.94 meters, the maximum error is 11.21 meters. The number of gross errors are 2 and the gross error rate is $8.3 \%$.

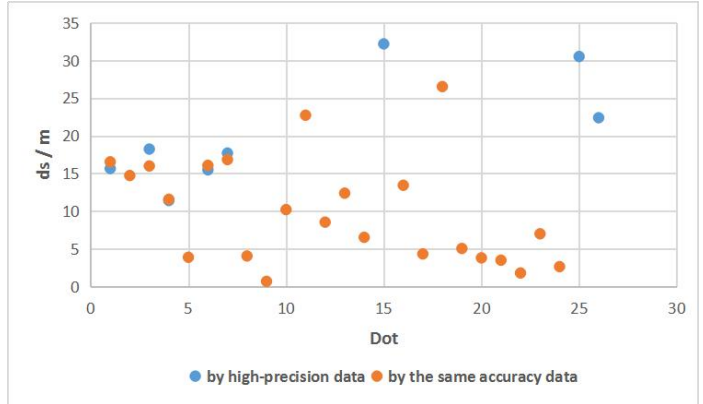

Figure 7. Errors of plane accuracy in field 3

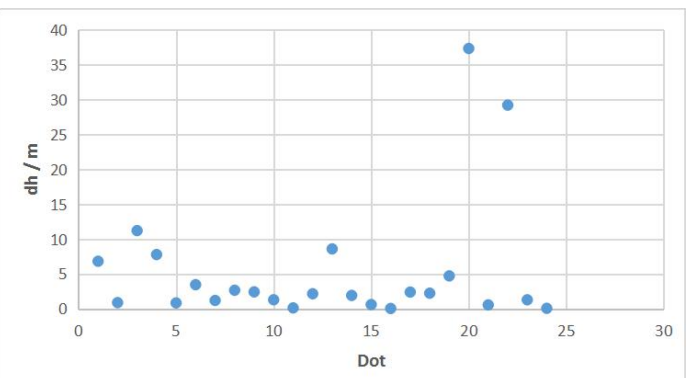

Figure 8. Errors of elevation accuracy in field 3

\begin{tabular}{|l|l|l|l|}
\hline & plane & plane & height \\
\hline $\begin{array}{l}\text { medium error } \\
\text { maximum error }\end{array}$ & 15.7 & 7.14 & 2.94 \\
$\begin{array}{l}\text { Number of gross } \\
\text { error }\end{array}$ & 3 & 16.25 & 11.21 \\
$\begin{array}{l}\text { Rate of gross } \\
\text { error } \\
\text { method }\end{array}$ & $37.5 \%$ & $8.7 \%$ & 2 \\
$\begin{array}{l}\text { high- } \\
\text { precision } \\
\text { data }\end{array}$ & $\begin{array}{l}\text { the same } \\
\text { accuracy data }\end{array}$ & $\begin{array}{l}\text { the same } \\
\text { accuracy data }\end{array}$ \\
\hline
\end{tabular}

Table 7. Statistical results of field 3

Field 4:There is no WorldView satellite imagery in field 4. Therefore, the plane accuracy and elevation accuracy of the test area were verified by the same accuracy.The statistical results are shown in Table 8.

The inspection results by using the same accuracy data show that the medium error is 2.26 meters in planimetric and 3.09 meters in height direction, maximum error is 5.57 meters in planimetric and 11.96 meters in height direction. The number of gross errors and the rates are both 0 . So, The accuracy of field 4 can meet the requirements of global project.

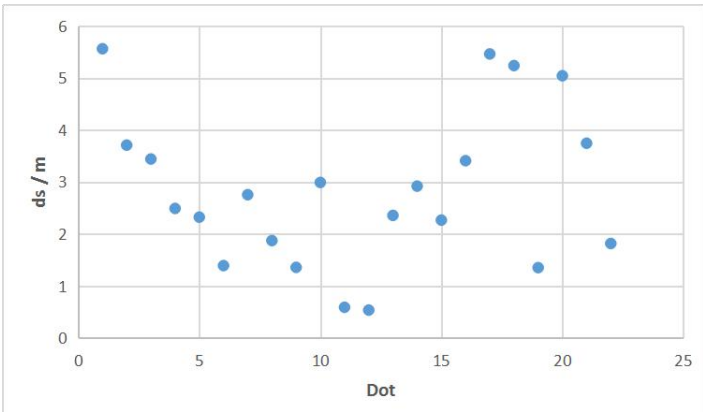

Figure 9. Errors of plane accuracy in field 4 


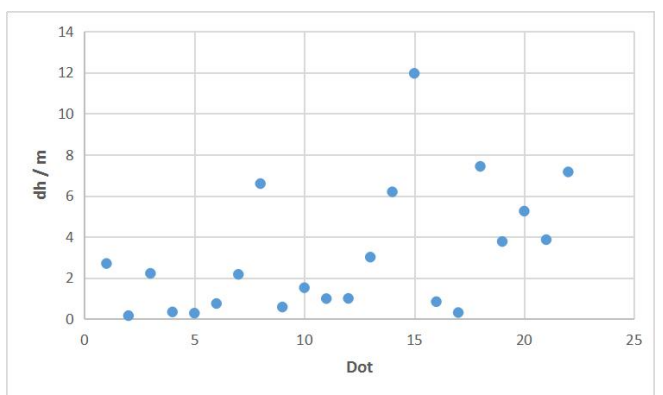

Figure 10. Errors of elevation accuracy in field 4

\begin{tabular}{|l|l|l|}
\hline & plane & height \\
\hline medium error & 2.26 & 3.09 \\
maximum error & 5.57 & 11.96 \\
$\begin{array}{l}\text { Number of gross } \\
\text { error }\end{array}$ & 0 & 0 \\
$\begin{array}{l}\text { Rate of gross error } \\
\text { method }\end{array}$ & 0 & 0 \\
\hline
\end{tabular}

Table 8. Statistical results of field 4

\section{CONCLUSION}

Through statistical analysis of the error values in the test area, and using the research methods proposed in this paper to check the test area, the plane accuracy and elevation accuracy of the field 1 , field 2 , field 4 can meet the project requirements. In the test area 3, the plane accuracy and elevation accuracy exceeded the requirements when using the high-precision data fixedpoint verification and the same accuracy data cross-validation inspection. When the tolerance of adjacent overlapping images within field 3 were checked, the result exceeded the accuracy requirements, too.

The inspection results are consistent that used the highprecision inspection data fixed-point verification, reference data cross-validation, and other software repeated calculation .At the same time, the inspection results of these three accuracy inspection methods are consistent with the results of relative orientation accuracy inspection and key technology inspection results.
Therefore, it can be determined that the inspection method can basically accurately reflect the position accuracy of the inspection area and it is possible to check the results area where the position accuracy has quality problems. This method can be used to check the position accuracy of global project results.

\section{REFERENCES}

Zhang Li, Zhang jixian, Chen Xiangyang, An Hong, 2009: Block-Adjustment with SPOT-5 Imagery and Sparse GCPs Based on RFM. Acta Geodaetica et Cartographica Sinica, 2009, 38(4): 302-310.

Zhou Ping, Tang Xinming, Cao Ning, Wang Xia, Li Guoyuan, Zhang Heng, 2016: SRTM-aided Stereo Image Block Adjustment withput Ground Control Points. Acta Geodaetica et Cartographica Sinica, 2016, 45(11): 1318-1327. DOI: 10.11947/j. AGCS. 2016. 20160219.

Gong Jianya, Wang Mi, Yang Bo, High-precision Geometric Processing Theory and Method of High-resolution Optical Remote Sensing Satellite Imagery without GCP. Acta Geodaetica et Cartographica Sinica, 2017, 46(10): 1255-1261. DOI:10. 11947/j. AGCS. 2017. 20170307.

Yang Bo, Wang Mi, Pi Yingdong. Block-adjustment without GCPs for Large-scale Regions only Based on the Virtual Control Points. Acta Geodaetica et Cartographica Sinica, 2017, 46(7): 874-881. DOI: 10.11947/j. AGCS. 2017. 20160588.

Wang Mi, Yang Bo, Li Deren, Gong Jianya, Pi Yingdong. Technologies and Applications of Block Adjustment Without Control for ZY-3 Images Covering China. Geomatics and Information Science of Wuhan University. 2017, 42(4), 427433. DOI: 10.13203/j. whugis20160534.

Cao Hui, Tao Pengjie, Li Haihong, et al. Using DEM as full controls in block adjustment of satellite imagery. Acta Geodaetica et Cartographica Sinica, 2020, 49(1): 79-91. DOI: 10.11947/j. AGCS. 2020.20190226. 